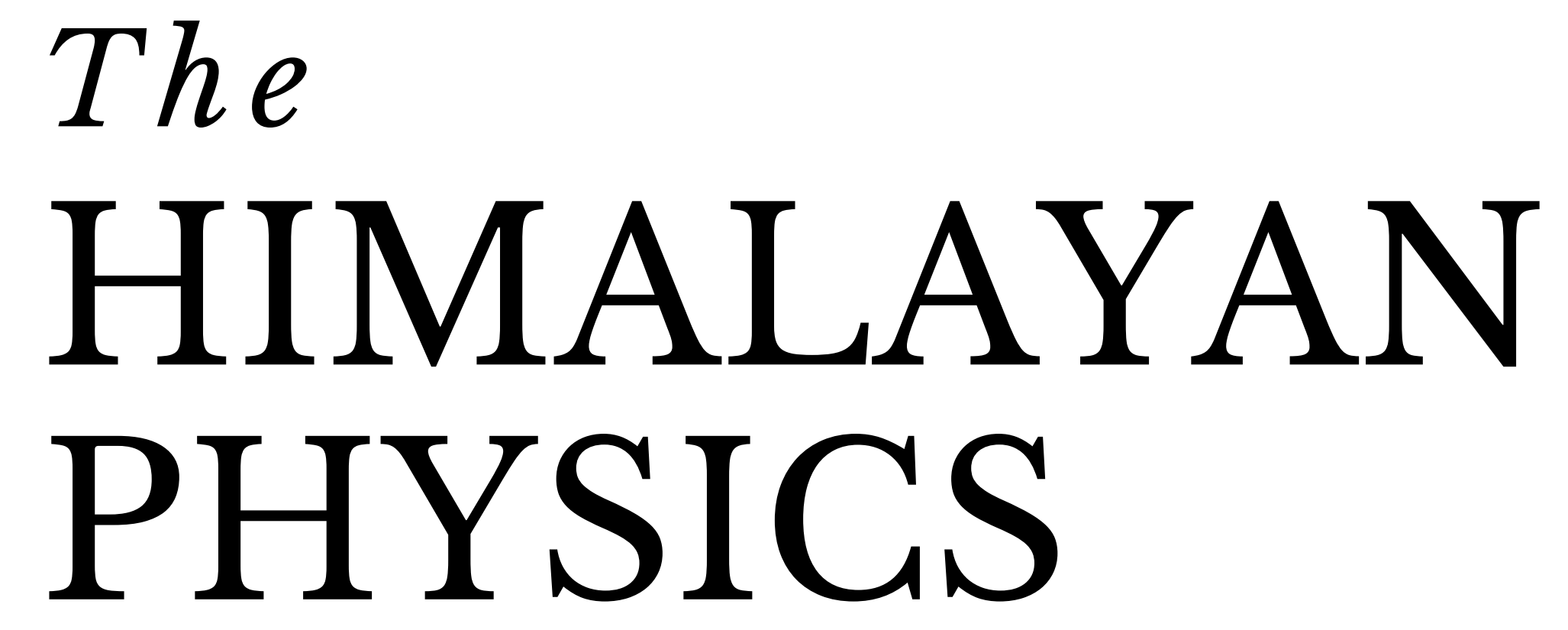

A peer-reviewed Journal of Physics

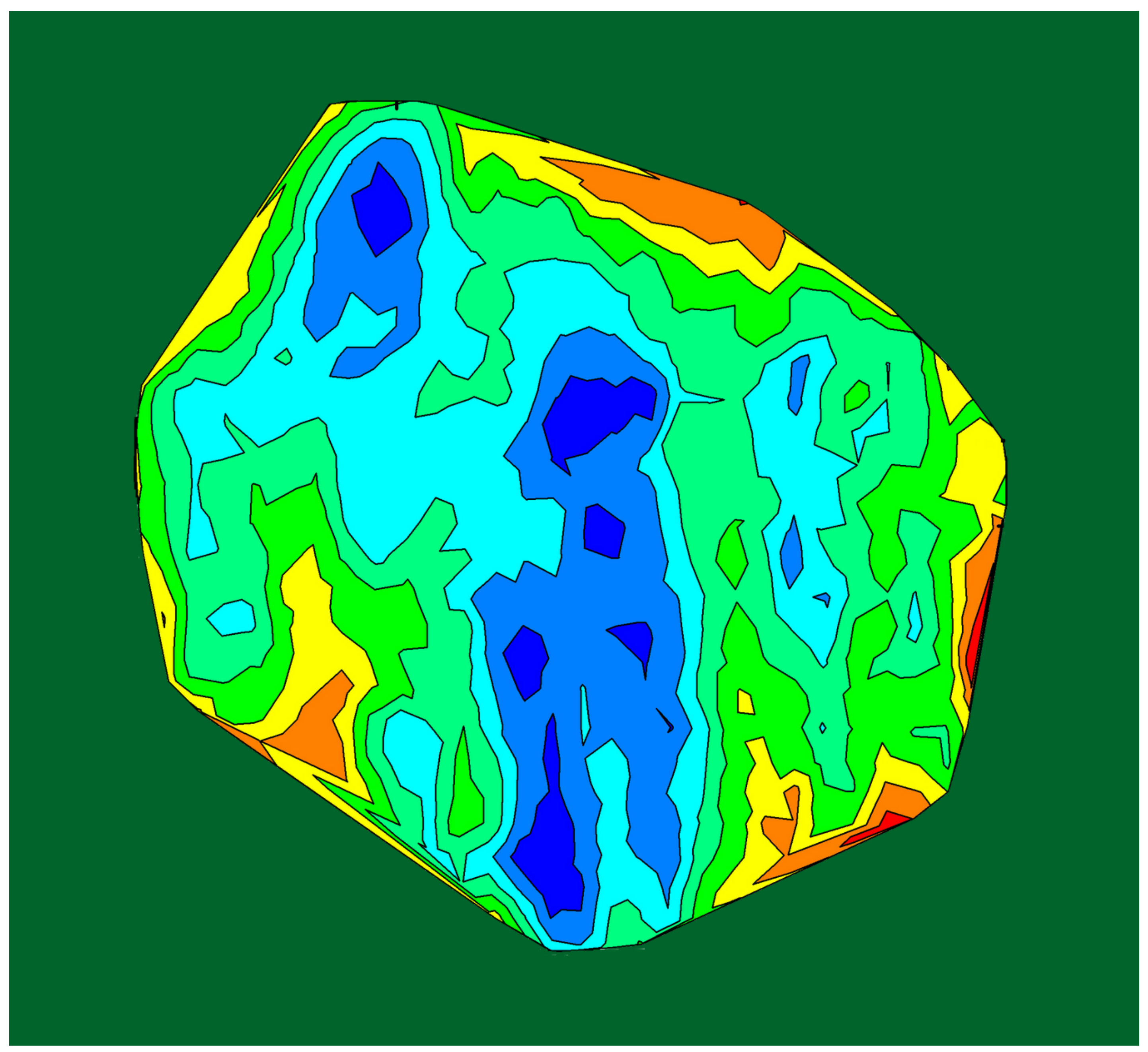

Department of Physics, Prithvi Narayan Campus, Pokhara Nepal Physical Society, Western Chapter, Pokhara 


\section{Publisher}

Department of Physics, Prithvinarayan Campus, Pokhara Nepal Physical Society, Western Chapter, Pokhara

\section{The Himalayan Physics}

Volume 9, December 2020

ISSN 2542-2545

The Himalayan Physics (HimPhys) is an open access peer-reviewed journal that publishes quality articles which make innovative contributions in all areas of Physics. HimPhys is published annually by Nepal Physical Society (Western Regional Chapter), and Department of Physics, Prithvi Narayan Campus, Pokhara. The goal of this journal is to bring together researchers and practitioners from academia in Nepal and abroad to focus on advanced techniques and explore new avenues in all areas of physical sciences and establishing new collaborations with physics community in Nepal.

\section{Chief Editor}

Kapil Adhikari

\section{Associate Editor \\ Aabiskar Bhusal}

(C)2020, Publishers. All rights reserved.

This publication is in copyright. Subject to statutory exception and to the provisions of relevant collective licensing agreements, no reproduction of any part may take place without written permission of the publishers.

Cover: Contour map of dust mass. (C) Mijas Tiwari. Printed from article in the current issue, with permission. 


\section{The HIMALAYAN PHYSICS}

A peer-reviewed Journal of Physics

\section{Chief Editor}

Kapil Adhikari

Associate Editor

Aabiskar Bhusal

\section{Publisher}

Department of Physics, Prithvi Narayan Campus, Pokhara Nepal Physical Society, Western Chapter, Pokhara 


\section{Nepal Physical Society Western Regional Chapter Pokhara, Nepal}

\section{President}

Min Raj Lamsal

Immediate Past President

Jeevan Regmi

Vice-President

Sundar Prasad Dhakal

Secretary

Ravi Karki

Treasurer

Dipak Adhikari

Joint Secretary

Sujan Lamsal

Editorial Member

Kapil Adhikari

Members

Amrit Dhakal

Laxman Thapa

Laxman Timilsina

Narayan Prasad Bhandari

Pradeep Subedi

Advisory Board

Prof. Dr. Pradip K. Bhattarai

Pabitra Mani Poudyal

Surya Bahadur G.C.

Parashu Ram Poudel

Prof. Dr. Shovakanta Lamichhane

Kul Prasad Dahal

Dr. Krishna Raj Adhikari

Ram Sajile Verma 


\section{Himalayan Physics Vol-9 (2020)}

\section{TABLE OF CONTENTS}

Metal Organic Frameworks(MOFs) as efficient carrier for targeted nanodrug delivery

R. Karki, D. Adhikari, K. Adhikari, N. Pantha

A Density Functional Theory Study on Paracetamol-Oxalic Acid Co-Crystal

P. Paudel, K.R. Adhikari, K. Adhikari

First-principles study of $\mathrm{C}$ cites vacancy defects in water adsorbed graphene

H.K. Neupane, N.P. Adhikari

Diusion of fructose in water: a molecular dynamics study

S. Bhusal, N. Pantha

Study of aecting factors of meteorological parameters on solar radiation on Pokhara P.M. Shrestha, J. Regmi, U. Joshi, K.N. Poudyal, N.P. Chapagain, I.B. Karki

Variation of mean value of velocity of ion with dierent obliqueness of magnetized plasma sheath

B.R. Adhikari, H.P. Lamichhane, R. Khanal

Study of dust properties of two far infrared cavities nearby asymptotic giant branch stars under infrared astronomical satellite maps

M. Tiwari, S.P. Gautam, A. Silwal, S. Subedi, A. Paudel, A. K. Jha

An experimental study on irradiated interface of silicon

M.R. Lamsal

Calculation of energy loss of proton beam on thyroid tumor

K. Giri, B. Paudel, B.R. Gautam

Study of noise level status at dierent rice mills in Surkhet Valley, Nepal

D.R. Paudel, H.N. Baral

Elliptically polarized laser assisted elastic electron-hydrogen atom collision and differential scattering cross-section

K. Yadav, S.P. Gupta, J.J. Nakarmi

Geodynamics of Gorkha earthquake (Mw 7.9) and its aftershocks

R.K. Tiwari and H. Paudyal 


\title{
Variation of mean value of velocity of ion with different obliqueness of magnetized plasma sheath
}

\section{Research Article}

\author{
B.R. Adhikari ${ }^{1 *}$, H. P. Lamichhane ${ }^{2}$, R. Khanal ${ }^{2}$ \\ 1 Department of Physics, Bhaktapur Multiple Campus, Tribhuvan University, Bhaktapur, Nepal \\ 2 Central Department of Physics, Tribhuvan University, Kirtipur, Kathmandu, Nepal
}

\begin{abstract}
Plasma sheath, which forms between a material wall and incoming plasma, plays an important role in controlling particle and energy fluxes to the wall. The problem of plasma sheath is one of the oldest in plasma and still draws attention, especially in magnetized plasmas. In this work velocity of ions in a magnetized plasma sheath has been studied using a kinetic trajectory simulation model for varying obliqueness of the field. Any change in obliqueness of the field causes the velocities to change. The change in mean value of the component normal to the wall is comparatively small whereas the other two components of velocity vary sinusoidally, nearly complementary to each other with nearly equal amplitudes.
\end{abstract}

Keywords: • Oblique magnetic field $\bullet$ Lorentz force $\bullet$ Plasma sheath $\bullet$ Mean value $\bullet$ Bohm criterionn

\section{Introduction}

Variation of mean value of ion velocity is recent field of research in plasma physics. It is obvious that the plasma interacts with the material surfaces and the proper understanding of this interaction is crucial in all plasma applications (e.g. plasma confinement for fusion, sputtering, etching, surface treatment, etc) [1-3]. Once the plasma-wall interaction is well understood it will be possible to control heat loading, energy transfer and particle flow towards the wall and hence the overall bulk plasma behavior [2]. In typical boundary layer problems a quasi-neutral plasma is shielded from a negative absorbing wall by a "thin" positive space charge region ("sheath") with a thickness of several electron Debye lengths $\lambda_{D}$. In the usual case, $\lambda_{D}<<L$, where $L$ is the characteristic extension of the plasma boundary layer. A sheath can only be formed, if the Bohm criterion $[4,5]$ is fulfilled.

The problem of plasma sheath is one of the oldest in plasma physics $[2,6,7]$ and the magnetized plasma sheath continues to receive a considerable amount of attention [8-12]. The problem of sheath formation at the plasma boundary is of importance for nearly all applications where a plasma is confined to a finite volume. Plasma

\footnotetext{
* Corresponding Author: b.r.adhikari@hotmail.com
} 
sheath has significant influence in charged particles and energy fluxes to the wall, which in turn considerably modifies the absorption, emission impurities and all other characteristics in the plasma. In this work we have studied the effect of obliqueness of the applied magnetic field various components of ion velocity in a magnetized plasma sheath using the kinetic trajectory simulation (KTS) Method.

\section{The KTS Method}

Most of the problems in plasma are studied using fluid theory, which is sufficiently accurate to describe majority of observed phenomena, however, for more accurate calculations, e.g., when sharp gradients are encountered the fluid treatment is inadequate. For these, we need to consider the velocity distribution function for each species and follow the kinetic treatment. KTS is an iterative method for numerically calculating self consistent, time independent kinetic plasma states in some given bounded spatial region [3, 12, 13] For given necessary initial and boundary conditions the distribution functions of the particle species are calculated by solving the related kinetic equations along the respective trajectories.

In the general case the Boltzmann equation for species-s is given as

$$
\frac{d f(\vec{x}, \vec{v}, t)}{d t}=\left[\frac{\partial}{\partial t}+\vec{v} \cdot \frac{\partial}{\partial \vec{x}}+\vec{a}^{s} \cdot \frac{\partial}{\partial \vec{v}}\right] f(\vec{x}, \vec{v}, t)=C^{s}
$$

with $\vec{a}^{s}(\vec{x}, \vec{v}, t)=\frac{q^{s}}{m^{s}}[\vec{E}(\vec{x}, t)+\vec{v} \times \vec{B}(\vec{x}, t)]$ where $\vec{E}(\vec{x}, t)$ and $\vec{B}(\vec{x}, t)$ are the macroscopic (i.e. locally averaged) electromagnetic fields, $\vec{a}^{s}$ is the macroscopic acceleration of species-s particles (i.e. it's acceleration in these fields), and $C^{s}$ is the species-s collision term. The kinetic Boltzmann equation (1) for collisionless cases takes the well known from of Vlasov equation:

$$
\left(\frac{\partial}{\partial t}+\vec{v} \cdot \frac{\partial}{\partial \vec{x}}+\vec{a}^{s} \cdot \frac{\partial}{\partial \vec{v}}\right) f(\vec{x}, \vec{v}, t)=0
$$

i.e., $f(\vec{x}, \vec{v}, t)=$ constant. This means that the velocity distribution function is constant for an observer moving along a collisionless trajectory.

\section{The Plasma Sheath Model}

The geometrical model of our magnetized plasma sheath is shown in Figure 1. The simulation region considered is bounded by two parallel planes situated at $x=0$ and $x=L$ and the plasma consists of only electrons and singly charged ions. The wall is at $x=0$ and the imaginary plane at $x=L$ is the "sheath entrance", which separates the non-neutral, collisionless sheath region $(x<L)$ from the quasineutral collisional presheath region $(x>L)$. 


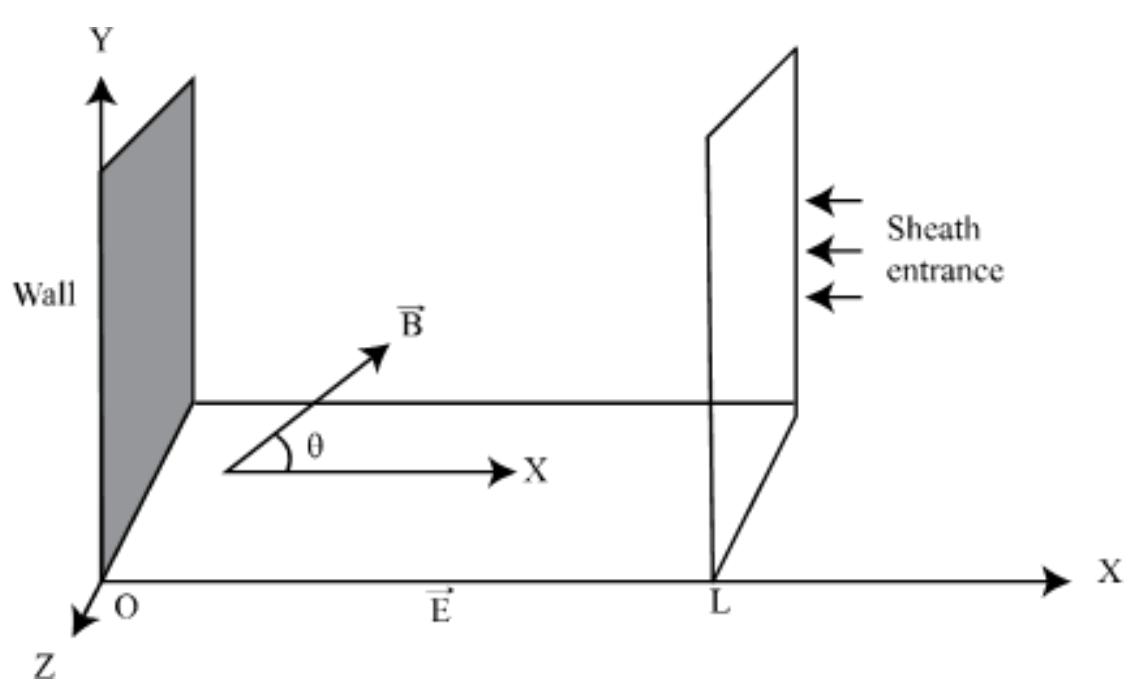

Figure 1. Geometry of the plasma sheath model

We consider a $1 d 3 v$ problem, i.e., the variation in plasma parameters is only along perpendicular to the wall (x-direction) whereas all three components of velocity are taken into account. This assumption is valid for studying a magnetized plasma sheath and is being used frequently [12]. The magnetic field lies in the $\mathrm{x}-\mathrm{y}$ plane where $\theta$ is the angle made by the oblique magnetic field with the $x$-axis, such that

$$
\vec{B}=B_{0}[\cos \theta \hat{x}+\sin \theta \hat{y}]
$$

The plasma particles (electrons and ions) enter the simulation region from the sheath entrance with cut-off Maxwellian velocity distribution functions, the wall doesn't emit any particles and both the boundaries are perfectly absorbing. Accordingly, the electron velocity distribution function is given by,

$$
f^{e}(x, v)=A^{e} \exp \left[-\left(\frac{v_{x}^{2}+v_{y}^{2}+v_{z}^{2}}{v_{t f}^{e^{2}}}\right)+\frac{e \phi(x)}{k T^{e}}\right] \Theta\left[v_{c}^{e}(x)-v_{x}\right]
$$

where $v_{c}^{e}(x)=\sqrt{\frac{2 e\left[\phi(x)-\phi_{0}\right]}{m^{e}}}$ is the electron cut-off velocity at $x, k$ is the Boltzmann constant and $\Theta$ is the Heaviside step function. The ion velocity distribution function at $x=L$ is given by,

$$
f^{i}(L, v)=A^{i} \exp \left[-\left(\frac{\left(v_{x}-v_{m L}^{i}\right)^{2}+v_{y}^{2}+v_{z}^{2}}{v_{t f}^{i^{2}}}\right)\right] \Theta\left(v_{c L}^{i}-v_{x}\right)
$$

where, $v_{t f}^{s}=\sqrt{\frac{2 k T^{s}}{m^{s}}}$ is the species-s (ion and electron) thermal velocity, $v_{m L}^{i}$ is the ion "Maxwellian-maximum" velocity at $x=L$ and $v_{c L}^{i}\left(v_{c L}^{i}<0\right)$ is the ion cut-off velocity at $x=L$. In the core plasma the particle distribution would obviously be Maxwellian, however, in case of sheath formation the ions are accelerated towards the wall so that they become shifted Maxwellian as given by equation (5). In addition, for the Bohm criterion to be satisfied 
by the ions they must have attained certain minimum velocity $\left(v_{c L}^{i}\right)$ at the sheath entrance. As the electrons are retracted and reflected by the negative potential wall their distribution gets cut-off at the sheath entrance as given by equation (4).

Different components of velocity of ions in the plasma sheath have been computed applying Lorentz force equation

$$
\vec{F}=q(\vec{v} \times \vec{B})+q \vec{E}
$$

For given initial and boundary conditions, we start with an initial guess to the potential profile and solve the governing equations (equation of motion, Poisson equation, Vlasov equation and Bohm-Chodura condition) iteratively until a self-consistent result is achieved $[12,13]$. We consider plasma parameters at the presheath by satisfying quasi-neutrality condition, the sheath- edge singularity condition, continuity of the first three moments of each species, and the Bohm- Chodura condition.

\section{Results and Discussion}

The variation of mean values of $x, y$ and $z$-component of velocity with respect to angles at constant magnetic field of $2.5 \mathrm{mT}$ are shown in Figures 2 to 5 . The mean value of $x$-component of velocity at magnetic field $2.5 \mathrm{mT}$ is very small but shows the oscillatory nature in the angular range from 0 to $90^{\circ}$. In the plot of mean value of $x$-component of velocity with respect to angle in the $5^{\circ}$ interval at magnetic field 2.5 mT, Figure 2 shows the minima at $10^{\circ}, 20^{\circ}, 40^{\circ}, 50^{\circ}$ and $75^{\circ}$. Similarly maxima of the velocity are found at $15^{\circ}, 25^{\circ}, 45^{\circ}, 60^{\circ}$ and $80^{\circ}$.

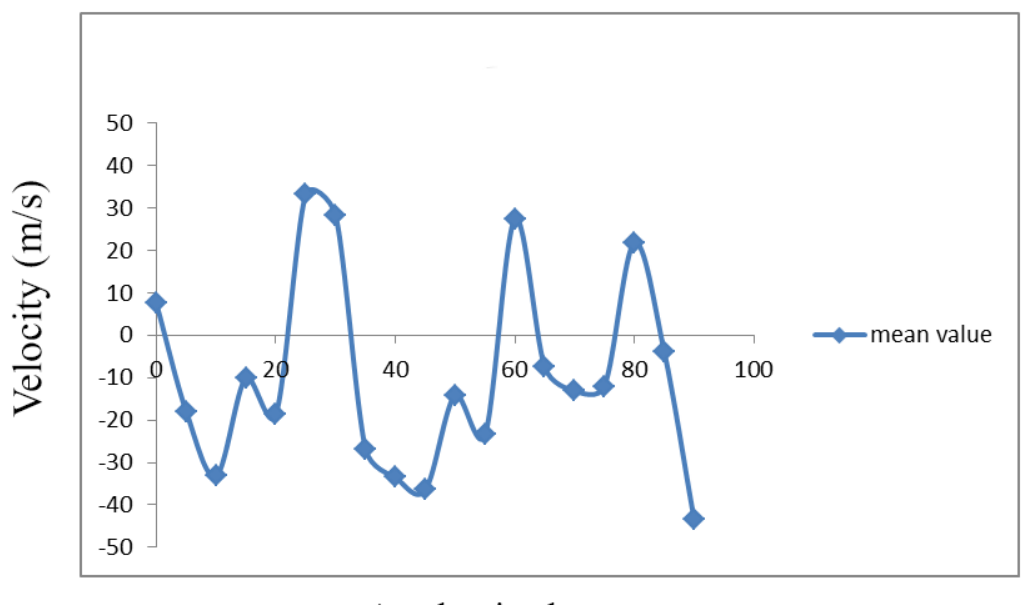

Angles in degree

Figure 2. Variation of mean values of $x$-component of velocity with respect to angles at magnetic field $2.5 \mathrm{mT}$

Figure 3 shows that the mean value of $y$-component of velocity is much more than that of $x$ - component. 
In the plot of mean value of $y$-component of velocity, as in Figure 3, the minima is found at angles $15^{\circ}, 25^{\circ}, 50^{\circ}$ and $75^{\circ}$. Similarly maxima of the velocity are found at $10^{\circ}, 20^{\circ}, 30^{\circ}, 45^{\circ}, 55^{\circ}, 70^{\circ}, 80^{\circ}$ and $90^{\circ}$. In the plot of mean value of $z$-component of velocity with respect to angles at magnetic field $2.5 \mathrm{mT}$, Figure 4 shows the minima at $5^{\circ}, 30^{\circ}, 40^{\circ}, 55^{\circ}, 65^{\circ}$ and $90^{\circ}$ whereas maxima of the velocity are found at $0^{\circ}, 10^{\circ}, 25^{\circ}, 35^{\circ}, 45^{\circ}, 60^{\circ}, 70^{\circ}$ and $85^{\circ}$

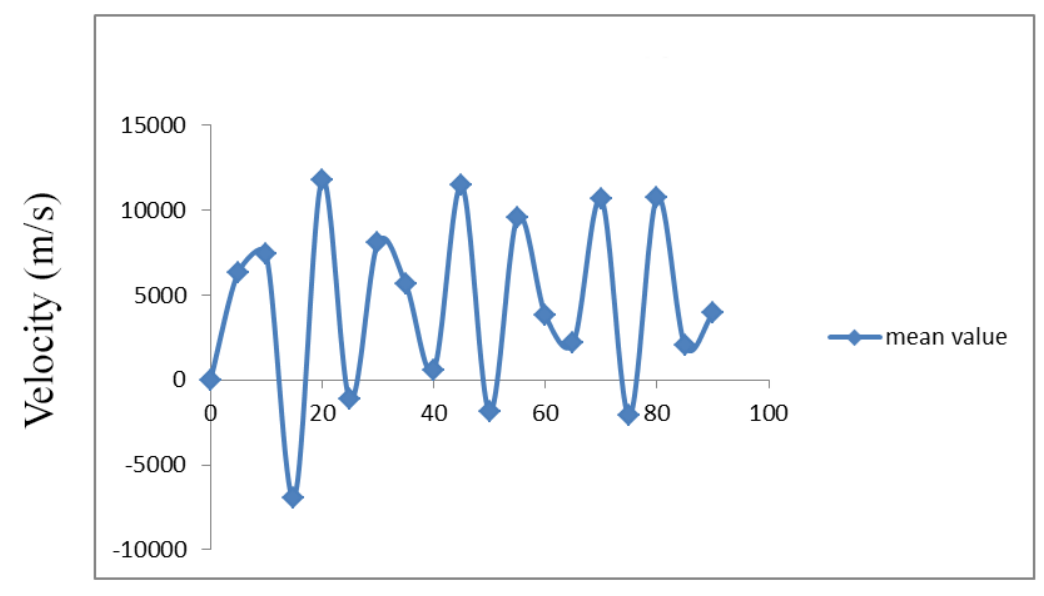

\section{Angles in degree}

Figure 3. Variation of mean values of $y$-component of velocity with respect to angles at magnetic field $2.5 \mathrm{mT}$

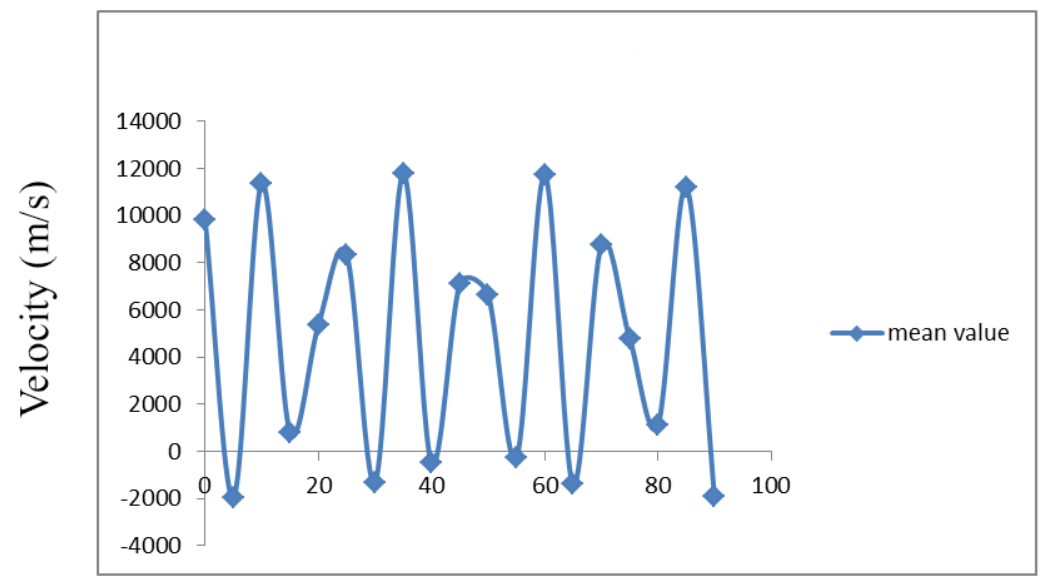

Angles in degree

Figure 4. Variation of mean values of $z$-component of velocity with respect to angles at magnetic field $2.5 \mathrm{mT}$ 
For easier comparison the variation of mean value of all the velocity components at different obliqueness are shown in Figure 5, which shows that among the minima the lowest value of mean value of $x$-component of velocity is around $-44 \mathrm{~m} / \mathrm{s}$ at $90^{\circ}$ whereas the maximum value is $33 \mathrm{~m} / \mathrm{s}$ at $25^{\circ}$. Similarly the lowest value and the maximum value of the mean value of $y$ - component of velocity is around $-6926 \mathrm{~m} / \mathrm{s}$ at $15^{\circ}$ and $11780 \mathrm{~m} / \mathrm{s}$ at $20^{\circ}$ respectively. Likewise the lowest and maximum value of the mean value of $\mathrm{z}$-component of velocity is around $-1949 \mathrm{~m} / \mathrm{s}$ at $5^{\circ}$ and $11710 \mathrm{~m} / \mathrm{s}$ at $60^{\circ}$ respectively.

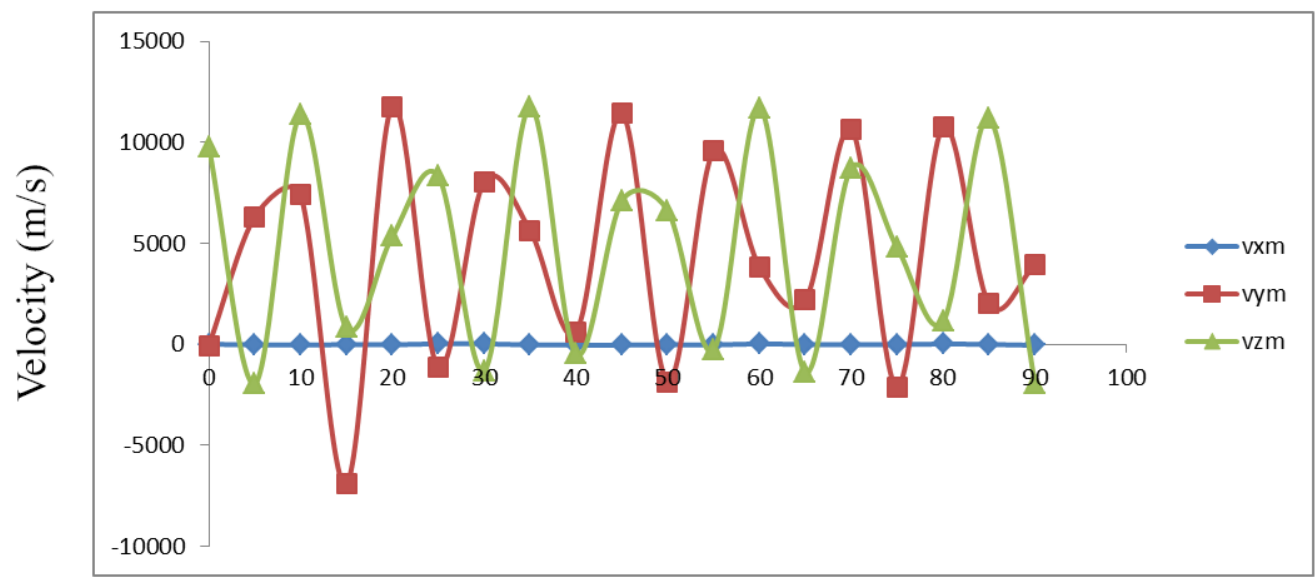

Angles in degree

Figure 5. Over all variation of mean values of velocity with respect to angles at magnetic field $2.5 \mathrm{mT}$

\section{Conclusions}

Ion velocity at presheath-sheath boundary is greatly affected by obliqueness of the field. As the obliqueness of the field changes the mean values of three component of the velocity also changes. The mean value of $x-$ component of velocity is comparatively small whereas $y$ and $z$-components of velocity vary sinusoidally, nearly complementary to each other with nearly equal amplitudes. This work provides a basis for studying all types of magnetized plasma sheath using kinetic approach and can be important in material processing, plasma etching, surface treatment, lighting, medicine and for confinement of plasma in fusion devices.

\section{References}

[1] Riemann KU. The Bohm criterion and sheath formation. Journal of Physics D: Applied Physics. $1991 ; 24(4): 493$.

[2] Riemann KU. Theory of the plasma-sheath transition. Journal of Technical Physics. 2000;41(1):89-121. 
[3] Chalise R, Khanal R. A kinetic trajectory simulation model for magnetized plasma sheath. Plasma Physics and Controlled Fusion. 2012;54(9):095006.

[4] Bohm D. Qualitative description of the arc plasma in a magnetic field. In: Guthrie A, Wakerling K R, editors. The characteristics of electrical discharges in magnetic fields. New York: McGraw- Hill; 1949. p. 1-12.

[5] Chodura R. Physics of Plasma-Wall Interactions in Controlled Fusion. Plenum Press, New York; 1986.

[6] Chodura R. Plasma-wall transition in an oblique magnetic field. The Physics of Fluids. 1982;25(9):1628-1633.

[7] Chen FF. Introduction to Plasma Physics and Controlled Fusion. 3rd ed. Plenum Press, New York; 2016.

[8] Moulick R, Goswami K. Sheath formation under collisional conditions in presence of dust. Physics of Plasmas. 2014;21(8):083702.

[9] Adhikari BR, Lamichhane HP, Khanal R. Temporal dependence of components of velocity of ions in a magnetized plasma sheath for different obliqueness of the magnetic field. In: Proceedings of 5th PSSI-plasma scholars colloquium.; 2016. .

[10] Chauhan S, Ranjan M, Bandyopadhyay M, Mukherjee S. Droplet shaped anode double layer and electron sheath formation in magnetically constricted anode. Physics of Plasmas. 2016;23(1):013502.

[11] Adhikari BR, Basnet S, Lamichhane HP, Khanal R. Presheath-sheath coupling for kinetic trajectory simulation of a magnetized plasma sheath. AIP Advances. 2019;9(5):055123.

[12] Regmi Y, Chalise R, Khanal R. Response of carbon and tungsten surfaces to hydrogen plasma of different temperatures. Physics of Plasmas. 2018;25(4):043521.

[13] Adhikari B, Basnet S, Lamichhane H, Khanal R. Beat frequency and velocity variation of ions in a magnetized plasma sheath for different obliqueness of the magnetic field. Journal of Institute of Science and Technology. 2018;23(1):88-92. 